\title{
A Short Proof of König's Matching Theorem
}

Romeo Rizzi

CWI

P.O. BOX 94079

1090 GB AMSTERDAM

THE NETHERLANDS

E-mail: romeo@cwi.n!

Received January 28, 1999; revised June 23, 1999

Abstract: We give a short proof of the following basic fact in matching theory: in a bipartite graph the maximum size of a matching equals the minimum size of a node cover. (c) 2000 John Wiley \& Sons, Inc. J Graph Theory 33: 138-139, 2000

Keywords: bipartite graph; maximum matching; minimum node cover

A matching of a graph $G(V, E)$ is a subset $M$ of $E$ such that every node of $G$ is incident with at most one edge in $M$. A cover of $G$ is a set of nodes $W$ such that $G \backslash W$ has no edges. Denote by $\nu(G)$ the maximum cardinality of a matching of $G$ and by $\tau(G)$ the minimum cardinality of a cover of $G$. Clearly, $\nu(G) \leq \tau(G)$.

We give a short proof of the following basic fact [1] in matching theory.

Theorem. Let $G$ be a bipartite graph. Then $\nu(G)=\tau(G)$.

Proof. Let $G$ be a minimal counterexample. Then $G$ is connected, is not a circuit, nor a path. So, $G$ has a node of degree at least 3. Let $u$ be such a node and $v$ one of its neighbors. If $\nu(G \backslash v)<\nu(G)$, then, by minimality, $G \backslash v$ has a cover $W^{\prime}$ with $\left|W^{\prime}\right|<\nu(G)$. Hence, $W^{\prime} \cup\{v\}$ is a cover of $G$ with cardinality $\nu(G)$ at most. Assume, therefore, there exists a maximum matching $M$ of $G$ having no edge incident at $v$. Let $f$ be an edge of $G \backslash M$ incident at $u$ but not at $v$. Let $W^{\prime}$ be

Contract grant sponsor: DONET PROJECT of the European Community.

Contract grant no.: TMR-DONET nr. ERB FMRX-CT98-0202.

c) 2000 John Wiley \& Sons, Inc. 
a cover of $G \backslash f$ with $\left|W^{\prime}\right|=\nu(G)$. Since no edge of $M$ is incident at $v$, it follows that $W^{\prime}$ does not contain $v$. So $W^{\prime}$ contains $u$ and is a cover of $G$.

The same proof easily extends to Egerváry's generalization [2] of König's result to graphs with nonnegative weights on the edges.

\section{References}

[1] D. König, Graphs and matrices, Mat Fiz Lapok 38 (1931), 116-119 (in Hungarian).

[2] E. Egerváry, On combinatorial properties of matrices, Mat Lapok 38 (1931), 16-28 (in Hungarian). 